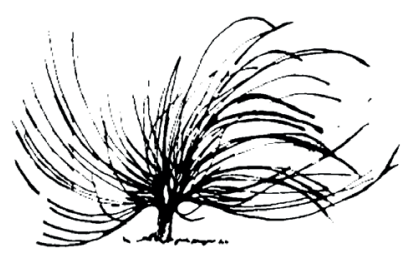

\title{
Adolescentes migrantes nicaragüenses en el sistema educativo costarricense: reflexiones desde y para la orientación
}

\author{
Cindy Artavia Aguilar ${ }^{1}$ \\ Universidad Nacional \\ Costa Rica \\ cindy.artavia.aguilar@una.cr
}

\begin{abstract}
Resumen
Este artículo surge de un proceso de investigación sobre el examen de la realidad educativa de ser migrante desde las experiencias socioeducativas de adolescentes migrantes nicaragüenses, con la finalidad de abrir espacios de reflexión y análisis, partiendo de la disciplina de orientación y su ámbito de acción en el sistema educativo. El estudio se realizó con diez personas adolescentes migrantes nicaragüenses en el sistema educativo costarricense. Los resultados de dicha investigación se socializaron y discutieron con estudiantes de la carrera de Orientación de la Universidad Nacional, con el propósito de establecer un espacio reflexivo a la luz de las posibles áreas de intervención desde la disciplina. Tales resultados giran en torno a tres categorías de análisis: la percepción de las repercusiones emocionales de ser estudiante migrante; toma de decisión y consecuencias de la migración, y, por último, los retos educativos de las personas migrantes. A partir de ellas, se establecen ideas conclusivas en permanente meditación, como la importancia reflexiva de los procesos de orientación,
\end{abstract}

Recibido: 23 de mayo de 2019. Aprobado: 28 de octubre de 2019.

http://dx.doi.org/10.15359/rep.15-1.10

1 Académica, investigadora de la Universidad Nacional. Licenciada en Orientación. Máster en Pedagogía con énfasis en Diversidad, Universidad Nacional. ORCID: http://orcid. org/0000-0002-2179-6808 
en torno a la diversidad y la interculturalidad desde la dignidad de la persona, y la potencialización tanto de habilidades como de destrezas para el desarrollo humano.

Palabras clave: migración, interculturalidad, orientación, sistema educativo costarricense, educación, equidad.

\begin{abstract}
This article arises from a research process on the analysis of the educational reality of being an immigrant from the social-educational experiences of Nicaraguan immigrant adolescents, in order to open spaces for reflection and analysis from the discipline of Counseling and its scope of action in the educational system. The study was carried out with ten Nicaraguan immigrant adolescents in the Costa Rican educational system and the results of the research were socialized and discussed with students of the Counseling major at Universidad Nacional, to establish a reflective space in light of possible areas of intervention from the discipline. These results come from three categories of analysis: The perception of being an immigrant student, decision-making and consequences of immigration, and finally, educational challenges from which conclusive ideas are established in a permanent reflection, such as the importance of a constant reflection on the processes of counseling around diversity and interculturality from human dignity, as well as the maximization of abilities and skills for human development.
\end{abstract}

Keywords: immigration, interculturality, Counseling, Costa Rican educational system, education, equity.

\title{
Introducción
}

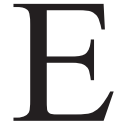

n los últimos años, Costa Rica ha experimentado un incremento en los porcentajes de personas que se movilizan dentro y fuera del país, además, enfrenta en su historia el flujo migratorio más alto, aspectos que no están al margen de la realidad mundial ni de las implicaciones en múltiples ámbitos y contextos sociales. En esta dinámica global de la migración, nuestro país afronta grandes retos y 
desafíos en educación, salud, trabajo, política y gobernanza, así como acarrea la responsabilidad de tomar acciones concretas en contextos determinados con poblaciones vulnerables.

Estos retos y desafíos están presentes en la realidad de la población costarricense y su diario convivir; por tal motivo, se resalta la importancia de visualizar los movimientos migratorios y su trascendencia en procesos de inclusión social en todos los ámbitos de acción de la humanidad. La educación, como pilar fundamental de la sociedad, debe abrir espacios de reflexión ante esta realidad y responder, en un marco de derechos humanos y aceptación a la diversidad, a la inclusión de las personas en condición migratoria en los procesos educativos.

Asimismo, la orientación como disciplina también requiere la reflexión-acción sobre sus procesos metodológicos-didácticos y los ámbitos personal-social, educativo y vocacional, en relación con las nuevas dinámicas sociales de migración; en tanto, nuestro país "podría situarse en torno a las 650 mil personas migrantes (tomando en cuenta la estimación de migración irregular), representado alrededor de un 13\% de la población total que habitan en Costa Rica" (DGME, 2017, p. 19).

A pesar de no ser un porcentaje tan alto en comparación con otros países de la región, hay que considerar que Costa Rica es una nación de alto tránsito migratorio y que el impacto social debe ser considerado en todos los ámbitos de acción del Estado, pues constituye un asunto importante para la vida nacional.

En educación, el fenómeno migratorio también debe ser considerado para el análisis de las nuevas realidades sociales y formativas de la población de niños y adolescentes; por ejemplo, en el Ministerio de Educación Pública, el 4,5\% son estudiantes extranjeros (37 788) para el año en curso (datos suministrados por el Departamento de Análisis Estadístico, MEP, 2019). Existen algunas investigaciones realizadas desde el Departamento de Investigación del MEP sobre interculturalidad, así como indagaciones generadas desde las universidades públicas; sin embargo, el tema de migración nicaragüense en el sistema educativo costarricense sigue siendo poco estudiado desde la teoría y las realidades expresadas por la población migrante nicaragüense. Más aún, toma relevancia social el considerar el sentir de la población adolescente y establecer dichos argumentos como punta angular de reflexión para la disciplina de la orientación en el sistema educativo. 
Por tal motivo, este artículo pretende aportar un espacio de análisis, a partir de información generada por medio de entrevistas a estudiantes de secundaria migrantes y datos sobre la realidad del contexto migratorio en Costa Rica.

\section{Marco teórico}

Es importante exponer algunas consideraciones teóricas que permitan ampliar el análisis de la realidad de las migraciones, la multiculturalidad, la interculturalidad y la relación con el sistema educativo costarricense.

\section{Migración}

Para el Alto Comisionado de las Naciones Unidas para los Refugiados (ACNUR) la definición de persona migrante y persona refugiada es diferente, Edwards (2016) indica que:

las personas migrantes eligen trasladarse no a causa de una amenaza directa de persecución o muerte, sino principalmente para mejorar sus vidas al encontrar trabajo o por educación, reunificación familiar, o por otras razones. A diferencia de los refugiados, quienes no pueden volver a su país de forma segura, los migrantes continúan recibiendo la protección de su gobierno (párr. 5).

Aunado a lo anterior, Arango (s. f.) (según se cita en Micolta, 2005) define las migraciones como: "desplazamientos o cambios de residencia a cierta distancia que debe ser significativa y con carácter relativamente permanente o con cierta voluntad de permanencia" (p. 60).

En el tema de las migraciones, se presenta un fenómeno que la literatura ha denominado duelo migratorio y obedece, entre muchas otras cosas, a los procesos de adaptación emocional que viven las personas migrantes a partir de la pérdida de aspectos sociales, familiares, emocionales, físicos, de regionalización, entre otros. Para Achotegui (2009, según se cita en Donoso, 2014) el duelo migratorio es

un duelo por muchas cosas, ya que posiblemente ninguna otra situación de la vida de una persona, incluso la pérdida de un ser querido supone tantos cambios como la migración. Todo lo que 
hay alrededor de la persona cambia, tanto más, cuanto más lejana y distante culturalmente sea la migración (p. 10).

\section{Características del duelo migratorio}

Para Donoso (2014, p. 12), la migración tiene características tales como las que se mencionan a continuación.

a. Es un duelo parcial, es decir, es más un duelo por separación, que, por pérdida, ya que lo que se deja sigue estando ahí, no desaparece, es más, se puede tener contacto con sus familiares y amigos, se puede saber lo que está pasando en su país, y hasta existe la posibilidad de visitarla temporalmente o de regresar en forma definitiva.

b. Es un duelo recurrente porque cada vez que la persona tiene contacto con su país, con sus familiares y amigos, inclusive cuando come algún plato típico o escucha música o el himno de su pueblo natal, vuelve a reavivar los vínculos.

c. Es un duelo vinculado a aspectos infantiles muy arraigados, ya que la persona que emigra está ya condicionada por lo que ha vivido en la infancia, el lugar en el que vivió, costumbres, tradiciones, paisajes, olores, sabores.

d. Es un duelo múltiple: porque se dejan muchas cosas, dentro de las cuales se puede mencionar la familia, la cultura, los amigos, la tierra, el estatus social.

Es importante considerar en cualquier proceso educativo el tema de duelo migratorio. Este permite prácticas pedagógicas más inclusivas y abre espacio al respeto de situaciones emocionales de las personas migrantes; como ya se mencionó, a las consecuencias del duelo por desarraigo de su patria y una separación ciertamente forzosa que involucra dejar la familia, las instituciones, amigos y costumbres.

\section{Diversidad}

Desde la diversidad presente en nuestra sociedad, y con la claridad de que vivimos en un mundo cada vez más complejo, cambiante y diverso, los sistemas educativos son espacios simbólicos de esta realidad y, por ende, las aulas en sus dinámicas formativas deben ser 
abiertas al cambio y generadoras de procesos de inclusión educativa. Arroyo (2013) menciona:

La gestión y la forma de entender la diversidad suponen el punto de partida en la educación inclusiva. En muchas ocasiones, las diferencias en nuestras escuelas sirven para justificar la exclusión educativa y, por ende, social de muchos niños y niñas. En especial, las de aquellos que tienen otras culturas y hablan una lengua diferente a la nuestra. Debemos precisar, no obstante, que la diversidad cultural del alumnado no ha surgido con la llegada de los niños de otros países, como muchos parecen pensar, recordemos el caso del alumnado de etnia gitana en España, durante mucho tiempo escolarizado en aulas puente. (Rodríguez Izquierdo, 2009; Ortiz Cobo, 2005; Navarro y Huguet, 2005). Como afirma Tuts (2007:34): "Las aulas no son ni han sido jamás homogéneas. La diversidad ha estado siempre presente en la sociedad." Debemos de ser conscientes de que el cambio al que se está llamando a la sociedad y a la escuela no viene sólo motivado por la llegada de personas de diferentes países y con diferentes lenguas, sino que estamos ante una situación de cambio global que provoca la necesidad de revisar los planteamientos del proceso de enseñanza y aprendizaje para responder a las nuevas necesidades sociales (pp. 145-146).

Partiendo de este principio, cualquier espacio educativo debe propiciar tanto la diversidad como el sustento de toda práctica de enseñanza-aprendizaje y considerar la necesidad de que el colectivo estudiantil y el docente sean conscientes de las desigualdades y, a su vez, puedan sensibilizarse ante la realidad diversa de los humanos. Al respecto, Arroyo (2013) cita:

Por tanto, la escuela debe fomentar la diversidad, pero debe combatir la que se convierte en desigualdad y atenta contra los principios de igualdad. Carbonell (2002) afirma que esta práctica debe asentarse en la "convicción de que somos mucho más iguales que distintos". De esta manera el principio de igualdad se convierte en un principio educativo tan importante como el de respeto a la diversidad (p. 147). 


\section{Pedagogía intercultural}

Para Leiva (2016), la pedagogía intercultural se plantea desde la necesidad de enriquecimiento cultural en los entes educativos en los cuales las personas se desenvuelven. Además, para que los procesos formativos, desde la pedagogía intercultural, encuentren acciones sustantivas en la reflexión-acción pedagógica, todas las personas involucradas en el contexto educativo toman un rol protagónico en la educación. Al respecto, dicho autor menciona:

Aprender del otro es algo bello, pero aprender de otro que además nos muestra referencias, códigos, costumbres, ideas y significados culturales diversos aporta a la escuela una enorme potencialidad de ser un auténtico espacio de humanidad, y por tanto, se requiere un enfoque inclusivo e interculturalidad para encontrar más que soluciones, nuevas preguntas de indagación didáctica sobre cómo afrontar el reto de vivir y convivir en una diversidad que nos envuelve y que puede ser maravillosa (p. 12).

La convivencia como un medio de crear interculturalidad, p a r a el estamento estudiantil y docente, adquiere mucho significado en el proceso de enseñanza-aprendizaje, en el marco de la pedagogía intercultural. Ortega (s. f., según se cita en Leiva, 2016) plantea algunos principios clave, los cuales serían:

1. Fomentar la promoción de la autonomía de los alumnos (...)

2. Comprender que los conflictos deben ser resueltos a través del diálogo para llegar o avanzar hacia un consenso, o hacia un pacto de respeto.

3. Potenciar aquellas situaciones problemáticas o conflictos para aprender a manifestarlos desde el respeto a los demás y el reconocimiento de la participación democrática (p. 32).

De igual forma, la comunicación intercultural es un mecanismo esencial en gestión y regulación de conflictos, en contextos formativos que presentan una gran diversidad cultural y esta no ha sido interiorizada como promotora de cambio social, educativo y personal. Algunas 
características de la comunicación intercultural en contextos culturalmente diversos son:

1. Integra competencias cognitivas y emotivas que permiten el establecimiento de relaciones positivas entre personas de procedencias culturales diversas.

2. Permite la toma de conciencia de la diversidad de orientaciones en la construcción de significados que den sentido a los conflictos interpersonales.

3. Valora las perspectivas y situaciones de diversidad indagando en un favor flexibilidad que atribuye un carácter más simétrico a las intenciones sociales.

4. Facilita la creación de un clima comunicativo cálido donde el diálogo es igualadamente importante al talante empleado, el tono del lenguaje, la expresión gestual y la afectividad en la relación (Leiva, 2016, pp. 32-33).

La comunicación intercultural en contextos multiculturales contribuye a generar procesos que coadyuvan en el bienestar integral del estudiantado, y a establecer vínculos sociales que permiten la construcción de espacios educativos más agradables, acogedores y de mayor significado para todas las personas involucradas en los procesos de enseñanza-aprendizaje.

\section{Orientación}

La orientación como disciplina promueve el bienestar integral de las personas en todas las etapas del ciclo vital y potencia las capacidades pedagógicas, psicológicas y socioemocionales de cada quien; todo lo anterior, con el propósito de generar una relación de armonía entre el desarrollo personal y el social.

La finalidad de la orientación es acompañar a todas las personas en el desarrollo de sus vidas y potenciar la prevención en todos sus niveles, como una posible estrategia de afrontamiento que permita la participación activa, crítica y transformadora en la sociedad.

\section{Ruta metodológica}

El estudio investigativo se desarrolló desde un enfoque cualitativo reflexivo y tiene como propósito analizar la realidad educativa de ser 
migrante, con base en las experiencias socioeducativas de adolescentes migrantes nicaragüenses, para abrir espacios de reflexión y análisis desde la disciplina de orientación y su ámbito de acción en el sistema educativo. De acuerdo con Clifford, (2001, según se cita en Apud, 2013), un enfoque cualitativo reflexivo es un texto inventivo polifónico que nos enfrenta a las complejidades de encuentros en el campo de investigación y nos permite tratar todas las reseñas textuales basadas en el trabajo de campo.

El estudio se realizó con una población de diez estudiantes adolescentes migrantes nicaragüenses, en diferentes colegios de Alajuela, durante el último trimestre lectivo del año 2018. Se aplicaron entrevistas a profundidad que giran en torno a tres categorías de análisis: 1) percepción de las repercusiones emocionales de ser estudiante migrante, 2) toma de decisión y consecuencias de la migración y, por último, 3) los restos educativos de las personas migrantes.

Una vez recogida la información y sistematizados los resultados, se estableció un espacio de análisis con estudiantes del curso de Orientación para la Diversidad de la carrera de Orientación de la Universidad Nacional, con la finalidad de discutir los resultados obtenidos a la luz de los posibles espacios de intervención desde la disciplina.

Por consideraciones éticas, se resguardan datos sociogeográficos y personales del colectivo participante y se cuenta con el consentimiento verbal de cada uno para ser parte de la investigación y publicación de resultados.

\section{Resultados y discusión}

Los resultados y la discusión surgen a partir de un grupo de preguntas generadoras que permiten el análisis de lo expuesto por el colectivo de adolescentes participantes en las entrevistas. Las preguntas generadoras fueron: ¿qué significa para usted ser migrante?, ¿qué situaciones influyeron en la toma de decisión para migrar del país?, ¿cuáles son las repercusiones personales de considerarse una persona migrante?, ¿cuál ha sido el mayor reto de adaptación a un nuevo país?, ¿qué apoyo considera importante en el nivel social o educativo debe tener un persona migrante desde el país de acogida? y ¿cuáles son las principales necesidades desde la población migrante? 


\section{¿Qué significa ser migrante?}

A partir de la consulta al grupo de estudiantes adolescentes sobre el significado de ser migrante, se obtuvieron las siguientes respuestas.

\section{Ser persona migrante...}

- Una persona migrante es aquella que deja su país para buscar mejores oportunidades y estabilidad laboral en otro país.

- Significa buscar una mejor vida, la cual en el país del que vivimos no se está tanto, por eso migrar es buscar mejores oportunidades que nos ayuden a salir adelante.

- Cuando las necesidades no se cubren en un país y los recursos son escasos se debe de buscar la manera de salir del país a otro que brinde una mejor estabilidad.

- Buscar mayor seguridad en un país donde no corra peligro de ser atacado por un ejército y morir.

- Dejar tus raíces, familia y amistades para comenzar de nuevo en un país diferente.

Cabe destacar que las frases citadas dejan entrever no solo el entramado de significados sociales y personales de lo que significa ser migrante para las personas, sino lo que representa para alguien adolescente quien se encuentra en un proceso de búsqueda de identidad propio de su edad y quien vive la migración como un cambio forzado y una ruptura abrupta en sus proyectos de vida.

La mitad de los adolescentes entrevistados se encontraba estudiando en su país de origen y señala el cambio hacia un nuevo país como una posibilidad de retomar sus estudios en el sistema educativo o de incorporarse a nuevos procesos formativos.

La "migración de corto recorrido" se visualiza como una opción de bienestar personal y familiar, la antesala a nuevas oportunidades de crecimiento personal, económico y social. Como menciona la Dirección General de Migración y Extranjería, en el Plan Nacional de Integración de Costa Rica 2018-2022:

Esta acentuación migratoria guardó relación con tres fenómenos estructurales que afectaron el istmo: a) el proceso de urbanización y modernización productiva en la región, b) la crisis producida 
por el conflicto armado y c) la transnacionalización económica y laboral como consecuencia de la globalización mundial (p. 10).

A pesar de que la migración se genera a partir de esos tres fenómenos mencionados, se sigue manteniendo la idea de que migrar para este grupo de personas es una posibilidad de superación personal y educativa.

\section{Situaciones que generan la toma de decisión de migrar}

En la tabla 1, se presentan frases que menciona el colectivo de adolescentes, en relación con el hecho que generó la toma de decisión familiar sobre la migración.

Tabla 1

Situaciones que generan la migración familiar

\begin{tabular}{|c|c|}
\hline \multirow[t]{2}{*}{ Conflicto interno } & $\begin{array}{l}\text { En Nicaragua se está dando una situación muy difícil } \\
\text { entre la población y el ejército por motivo de la } \\
\text { presidencia que este posee, hay muchas matanzas y } \\
\text { las vidas de nosotros los nicaragüenses corren peligro, } \\
\text { por ese motivo tuvimos que migrar de manera ilegal a } \\
\text { Costa Rica. }\end{array}$ \\
\hline & $\begin{array}{l}\text { Migramos porque sentíamos que corríamos peligro, al } \\
\text { ver cuántas personas morían y está propenso uno a ser } \\
\text { víctima también. }\end{array}$ \\
\hline \multirow[t]{6}{*}{$\begin{array}{l}\text { Situación } \\
\text { socioeconómica }\end{array}$} & $\begin{array}{l}\text { Debíamos salvar la vida de nosotros, no queríamos que } \\
\text { por culpa del Gobierno nos pasará algo malo, además } \\
\text { de que casi no teníamos qué comer y la necesidad y } \\
\text { limitación de recursos era muy pocos. }\end{array}$ \\
\hline & $\begin{array}{l}\text { Decidimos migrar porque en Nicaragua no tenía trabajo } \\
\text { y no podíamos comprar casi ni para comer. }\end{array}$ \\
\hline & $\begin{array}{l}\text { Decidimos migrar, por falta de recursos económicos en } \\
\text { Nicaragua y porque contaba con apoyo de familiares en } \\
\text { Costa Rica. }\end{array}$ \\
\hline & $\begin{array}{l}\text { Para salir de la pobreza que vivía en Nicaragua, donde } \\
\text { no había ni qué comer muchas veces. }\end{array}$ \\
\hline & $\begin{array}{l}\text { La falta de trabajo era mucha, por lo tanto, decidimos } \\
\text { venir a Costa Rica en busca de trabajo. }\end{array}$ \\
\hline & $\begin{array}{l}\text { La economía del país nicaragüense está muy mal, por } \\
\text { lo tanto, se debe migrar a otro país en busca de mejores } \\
\text { oportunidades. }\end{array}$ \\
\hline
\end{tabular}


Situaciones que generan la migración familiar

\begin{tabular}{ll}
\hline Unificación & $\begin{array}{l}\text { Decidimos venirnos porque tenemos familiares aquí en } \\
\text { familiar }\end{array}$ \\
& $\begin{array}{l}\text { Costa Rica, y porque aquí hay mejores oportunidades } \\
\text { para trabajar lo que nos ayuda a tener un sueldo que nos } \\
\text { permita comprar la comida para sobrevivir. }\end{array}$ \\
\cline { 2 - 2 } & $\begin{array}{l}\text { Migramos a Costa Rica porque mi papá es costarricense } \\
\text { y debíamos viajar para establecer nuestra familia aquí, } \\
\text { ya que él tenía su trabajo en este país. }\end{array}$
\end{tabular}

Nota: Elaboración propia.

Existen muchos factores que promueven la movilización migratoria, a como se observa en la tabla 1; sin embargo, en el caso de las personas nicaragüenses, se consideran como principales factores los que están asociados a la realidad del istmo (los ya nombrados) y también el Plan Nacional de Integración de Costa Rica 2018-2022 menciona:

Entre los factores explicativos de la migración reciente de nicaragüenses hacia Costa Rica se pueden señalar los siguientes: la insuficiencia del mercado laboral nicaragüense para absorber toda la mano de obra con que cuenta; las diferencias de salario entre ambos mercados laborales y la cercanía geográfica de ambos países; la ocurrencia de eventos naturales devastadores; la reunificación familiar; o las expectativas de aumentar el acceso a las oportunidades (Consejo Nacional de Migración, 2013, según se cita en Dirección General de Migración y Extranjería, 2019, p. 10).

El conflicto interno, la situación socioeconómica y la unificación familiar son las principales razones en la toma de decisión para la migración. Es importante destacar que estos fenómenos son llevados a las aulas educativas, desde las experiencias personales del estudiantado, $\mathrm{y}$, por ende, están inmersos en las dinámicas educativas grupales, así como en la atención individual de cada estudiante. 

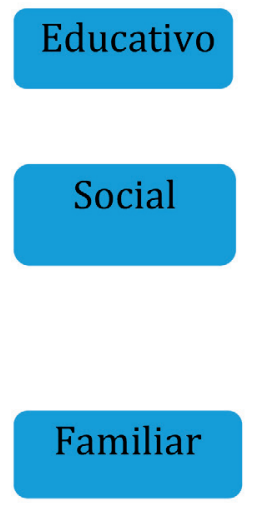

\section{Emocional} Dejar mis estudios en Nicaragua y no poder continuarlos en Costa Rica, por ser
indocumentado y tener que trabajar para apoyar a mi familia.

Que mi familia no puede recibir atención médica cuando están enfermos, porque no

No tener control de situaciones de salud de la familia, todos sufrimos por eso, el dinero no alcanza para esas cosas y mi abuela está muy enferma.

Vivir en una casa prestada en malas condiciones y condiciones deplorables.

Sobrevivir con un sueldo bajo, que no cubre seguro social para la atención médica.

Acostumbrarme a un lugar diferente, acomodarme a la cultura y creencias costarricenses que son diferentes a las mías.

No ver la familia por varios meses, luego de ser muy unidos cuando estábamos en Nicaragua.

Abandonar a mi familia, separándome de esta y teniendo comunicación por ocasiones con estos, lo que trae la separación familiar.

No ver a mis abuelos por mucho tiempo, con la preocupación de la situación que está pasando en Nicaragua.

Figura 1. Consecuencias de las migraciones.

Nota: Elaboración propia.

Huirle constantemente a la ley, por ser indocumentados, por eso vivimos con miedo de salir, porque nos pueden deportar.

No me siento igual, todo cambió de repente, muchas cosas nuevas.

Es muy difícil, no me ven bien, y creo que tienen razón.

Las consecuencias son múltiples; tal y como se observa en la figura 1, estas emociones, sentimientos y realidades son parte del desarrollo socioemocional y educativo del estudiantado. Todo está entrelazado en el desenvolvimiento de experiencias de aprendizaje y, por tanto, la importancia de retomar, en los procesos educativos y, sobre todo, en procedimientos de orientación, todos aquellos factores socioemocionales que pueden incidir en el desarrollo personal-educativo y social del individuo. La UNESCO (2018), en el Informe de Seguimiento de la Educación para el Mundo 2019, presenta como una recomendación la atención a las necesidades educativas de las personas migrantes y desplazadas, en el marco de la ayuda humanitaria y para el desarrollo; así, cita: "Para satisfacer las necesidades, se debe multiplicar por diez el porcentaje dedicado a la educación en la ayuda humanitaria" (p. 73).

En el caso de la orientación en Costa Rica, la atención integral desde los servicios que brindan los departamentos de esta rama del conocimiento en los centros educativos está fundamentada desde la equidad y la inclusión educativa-social, en un marco de derechos 
humanos y para la paz. Por ello, el llamado a que se revise y reflexione, desde las acciones personales y profesionales, acerca del tipo de atención brindada en todas las áreas del desarrollo humano y partiendo de las diversas modalidades de intervención de la disciplina en el ámbito educativo. Asimismo, es fundamental el acompañamiento en los procesos educativos institucionales y las redes de apoyo que se puedan establecer para la población en general, desde el tema de las migraciones nicaragüenses en Costa Rica.

\section{Retos educativos de las personas migrantes}

Los retos son múltiples en el tema de las migraciones, más cuando hablamos de poblaciones de jóvenes estudiantes, que presentan muchas luchas en cuanto a permanencia en el sistema educativo, aprobación, rendimiento educativo, estabilidad socioemocional, desarrollo de habilidades para la vida, entre otros.

El grupo de estudiantes menciona como principales retos los siguientes:

- El mayor reto es adaptarme a no contar con ningún servicio público, ya sea salud o educación, por estar de ilegal.

- Un reto ha sido no recibir ninguna ayuda económica por instituciones o familiares, y no tener ninguna documentación.

- Dejar parte de mis amigos en Nicaragua, sabiendo lo que está pasando en mi país actualmente; todo aquí en el colegio es diferente, a veces no entiendo nada.

- A mí me cuesta estudiar, y aquí es más difícil, es como ser algo extraño.

- Un obstáculo ha sido que mi familia no tiene trabajo fijo, sino que dependemos de trabajos que salen de momento, lo cual implica no tener un salario fijo, para satisfacer mis necesidades y las de mi familia.

- Adaptarse a una cultura diferente, a comidas y clima al que no estaba acostumbrada.

- Primer reto fue ingresar al país indocumentado, luego no contar con atención médica en caso de enfermedad y entrar a estudiar es muy difícil.

- Dejar los estudios, lo cual me gusta hacer para un mejor futuro, pero debido a la situación académica, ahora solo trabajo y estudiar se me imposibilita y ha sido un reto porque pasé de estudiar a trabajar en labores muy difíciles o que requieren fuerza.

- Salir adelante, sin el apoyo cercano de amistades o personas significativas en mi vida. 
Cada uno de los retos expresados entreteje un entramado social complejo desde las propias realidades y los recursos sociales, culturales económicos, familiares y educativos existentes, con lo que cada joven hace frente al sistema educativo; asimismo, se priorizan necesidades básicas, muchas no resueltas, como alimentación, vivienda, salud y familia, antes de cualquier proceso de aprendizaje institucionalizado.

Los principales retos dentro y fuera del sistema educativo están en brindar apoyo cada vez más, inclusive, que se permita un acceso real a la formación y desde el cumplimiento de derechos humanos, con enfoque de equidad. Como menciona Luis Almagro, secretario general de la Organización de los Estados Americanos (OEA), «la promoción de "más derechos para más gente"》 (OEA. 2016, p. 7).

Al respecto, el colectivo estudiantil entrevistado menciona algunos obstáculos que limitan su acceso o permanencia en centros educativos:

1. No contar con documentos que lo identifiquen en Costa Rica.

2. Falta de atención médica por no tener seguro social.

3. Vivir en casas con condiciones insalubres.

4. No tener beca para estudiar en las escuelas costarricenses.

5. No disfrutar de ayuda socioeconómica de instituciones para poder cubrir sus necesidades básicas.

6. Salarios por debajo de lo establecido para un agricultor o jornalero.

7. Poca participación en actividades sociales en la comunidad.

Los hechos mencionados, si bien es cierto algunos no obedecen a situaciones propias del sistema educativo o que se pueden solventar desde este ámbito, son limitaciones sociales- culturales no resueltas y evidencian la necesidad de políticas públicas formativas y sociales orientadas una educación más equitativa.

Es imperante que se abran aún más los espacios de reflexión sobre las realidades de las personas migrantes en el sistema educativo formal y que resultados de investigaciones en esta temática permitan ahondar la discusión e incidir en el currículum nacional, en la transformación de prácticas pedagógicas más inclusivas y conscientes del sentir-pensar de los grupos culturalmente diversos. Las percepciones que tienen los migrantes, al igual que, por ende, la toma de decisiones 
y cambios en los niveles personal, social y educativo transforman espacios privados de vida en nuevos escenarios educativos y sociales. Las demandas presentes y que se avecinan plantean retos educativos novedosos en sitios concretos como las aulas y estas, a su vez, solicitan más formas de organización, planeamiento y acciones pedagógicas.

\section{Ideas conclusivas y en permanente reflexión}

Es importante destacar la realidad de las migraciones, así como su influencia en los procesos educativos y, muy especialmente, en los servicios de orientación desde nuestro sistema educativo. A modo de conclusiones que permanecen en constante construcción, se puede mencionar:

- La visualización de una realidad presente en nuestra sociedad y en nuestras aulas, que no puede ser ajena a los procesos de educación nacional, la toma de decisiones en políticas educativas y la construcción de nuevos escenarios pedagógicos.

- La deconstrucción y construcción de redes de apoyo fundamentadas en equidad y derechos humanos, que garanticen inclusión educativa y permitan abrir espacios de participación real y democrática en educación.

- El análisis desde las acciones afirmativas en educación para otras concretas en los procesos de admisión, permanencia y rendimiento educativo, que garanticen un sistema educativo, el cual respete los derechos humanos, los promueva y los viva en todos los procedimientos.

- La reflexión constante de los procesos de orientación en torno a la diversidad y el estímulo al diálogo digno intercultural, desde la dignidad de la persona; la potencialización de habilidades y destrezas para el desarrollo humano, desde el círculo de bienestar integral y la prevención.

- Los objetivos de la investigación se cumplen, al evidenciar las necesidades de la población migrante nicaragüense en el sistema educativo. No se puede omitir dichas realidades ni ser negligente en la atención educativa que requieren las poblaciones estudiantiles culturalmente diversas. 


\section{Referencias}

Apud, I. (2013). Repensar el método etnográfico. Hacia una etnografía multitécnica, relfexiva y abierta al diálogo interdisciplinario. Revista Antropología Arqueológica, 16, 213-235. Recuperado de https://revistas.uniandes.edu.co/doi/pdf/10.7440/ antipoda16.2013.10

Arroyo, M. (2013). La Educación Intercultural: un camino hacia la inclusión educativa. Revista de Educación Inclusiva, 6(2), 144-159. Recuperado de https://dialnet.unirioja.es/servlet/ articulo? codigo $=4335836$

Dirección General de Migración y Extranjería (DGME). (2017). Diagnóstico del Contexto Migratorio de Costa Rica 2017. San José, Costa Rica: DGME.

Dirección General de Migración y Extranjería (DGME). (2019). Plan Nacional de Integración para Costa Rica 2018-2022. San José: DGME.

Donoso, G. (2014). El duelo migratorio. Recuperado de http://www. tanatologia-amtac.com/descargas/tesinas/136\%20Duelo\%20migratorio.pdf

Edwards. A. (2016). ¿Refugiado o migrante? ¿Cuál es el término correcto? United Nations High Commissioner for refugees. Recuperado de https://www.acnur.org/noticias/noticia/2016/7/5b9008e74/ refugiado-o-migrante-cual-es-el-termino-correcto.html

Leiva, J. (2016). Abriendo caminos de interculturalidad e inclusión en la escuela. Madrid, España: Editorial DYKINSON, S. L.

Micolta, A. (2005). Teorías y conceptos asociados al estudio de las migraciones internacionales. Trabajo social, 7, 59-76. Recuperado de https://dialnet.unirioja.es/servlet/articulo? $\operatorname{codigo}=4391739$

Organización de los Estados Americanos (OEA). (2016). Equidad e Inclusión social: superando desigualdades hacia sociedades más inclusivas. Washington: Departamento de Inclusión Social, Secretaría de Acceso a Derechos y Equidad, OEA.

UNESCO. (2018). Resumen del Informe de seguimiento de la educación en el mundo 2019: Migración, desplazamiento y educación: Construyendo puentes, no muros. París: UNESCO. 
